\title{
A high performance integrated readout circuit for wavefront sensors
}

\author{
Pablo N. Agra Belmonte ${ }^{1}$ Ursula V. Abecassis ${ }^{1,3} \cdot$ Lucas Chaves $^{2} \cdot$ Luciana P. Salles $^{1,2}$. \\ Davies W. de Lima Monteiro ${ }^{1,2}$ (1)
}

Received: 10 December 2019 / Accepted: 29 September 2020 / Published online: 13 October 2020

(c) Springer Nature Switzerland AG 2020

\begin{abstract}
This work presents an integrated pixel topology that promises to offer superior performance in a Hartmann-Shack Wavefront Sensor (WFS) with an orthogonal array of Quad Cells serving as Position-Sensitive Detectors. The readout integrated circuit for each photodiode is fully compliant to any standard CMOS microelectronics technology and is advantageously tolerant to high background illumination levels whereas maintaining both high linearity and high sensitivity. To assess the operation of this pixel on the focal-plane array of the WFS, we developed a computational platform encompassing a full detection chain comprising wavefront sampling, photodetection, electronic circuitry and wavefront reconstruction. It couples an algorithm written in C to SPICE (Simulated Program with Integrated Circuits Emphasis). The platform is technology agnostic and flexible, enabling easy modification to represent different detection or reconstruction methods. The results obtained with the proposed pixel have been compared to those obtained with a conventional pixel in CMOS image sensor.
\end{abstract}

Keywords Wavefront sensor · Pixel · Microelectronics · Integrated circuit · Quadrant detector · Wavefront sensing · Hartmann-Shack

\section{Introduction}

Wavefront Sensors (WFS) are consolidated and ubiquitous in Adaptive Optical (AO) systems. Among all types of WFS, the Hartmann-Shack (HS) method is one of the most employed, being versatile and rather simple [1,2]. It features robustness to vibrations; compact assembly; relative low cost; flexible adaptation to different magnitudes of aberrations; and fast WF reconstruction [3]. There have been several adaptive optical system open-access simulation tools implemented, mostly targeting astronomy, but with a potential for adaptation to other fields [4-12]. Although developed to different degrees of completeness and universality, they do not yet offer the possibility to couple electronic-circuit simulations, on circuit and device level, along with optical and data-processing algorithms. In this work, we evaluate the impact of a promising integrated-circuit pixel topology at the focal-plane array of a HS WFS by means of a computational platform we developed for the seamless simulation of the optical and electronic signal flow, whose details are presented in previous works $[13,14]$.

The platform includes the modelling of the input beam and aberration; the microlens array; the Position-Sensitive Detectors (PSDs); the readout integrated circuits (ROICs); and a modal WF reconstruction algorithm. It conveniently enables the evaluation of different topologies and circuits prior to the fabrication of the device. The main focus of the paper is to assess the performance of a superior integrated pixel topology at the focal plane of the sensor on

$\triangle$ Davies W. de Lima Monteiro, davies@ufmg.br| ${ }^{1}$ Graduate Program in Electrical Engineering, Universidade Federal de Minas Gerais (UFMG), Belo Horizonte, Brazil. ²Department of Electrical Engineering, UFMG, Av. Antonio Carlos, 6627 - Pampulha, Belo Horizonte, MG 31270-010, Brazil. ${ }^{3}$ Department of Electronics and Telecommunications, Instituto Federal do Amazonas, Av. Governador Danilo Areosa, 1672- Distrito Industrial, Manaus, AM 69075-351, Brazil. 
the wavefront reconstruction error, comparing it to the results when deploying a pixel conventionally used in image sensors.

The focal plane of the HS WFS in this work consists of an orthogonal array of quad-cells (QCs) serving as position-sensitive detectors (PSDs). The readout integrated circuit (ROIC) connected to each photodiode of a QC was simulated for a standard AMS $0.35 \mu \mathrm{m}$ CMOS technology and its main function is to convert the photocurrents into voltage signals over a predetermined integration period. These signals are subsequently used to calculate the coordinates of the sampled light spots, serving as inputs to the WF reconstruction algorithm. This paper assesses the WFS simulation results for a ROIC based on the Bouncing Pixel topology, previously developed by us $[15,16]$. As a reference, an enhanced version of the conventional and widely used Active-Pixel-Sensor (APS) is also simulated [17]. In this version, the reset transistor is a PMOS, increasing its voltage swing, and it is equipped with an analog transmission gate between the photodiode and the gate of the sourcefollower node, to allow global shutter operation, in which all pixels can be read simultaneously, avoiding artefacts in dynamic wavefront sampling due to sequential time readings. In comparison to the APS, the Bouncing PIxel has the ability to prevent signal saturation, yet featuring high linearity and high sensitivity, in response to variations in light intensity, therefore accommodating a wider range of photocurrents. This means HDR (High Dynamic Range) operation, as regards light-intensity range. The spot-position dynamic range, usually considered in WFS analyses, refers to how far a light spot can be displaced on the focal plane and is not affected by the circuit choice. This pixel can be designed to be integrated either in image-sensor arrays or alternatively in custom PSD arrays. The results show a better quality of WF reconstruction with much lower RMS errors, demonstrating that this circuit can be a suitable choice in WFS focal-plane design.

A HS WFS is composed of an array of microlenses, whose purpose is to sample patches of the input WF, as shown in Fig. 1a, depicting one specific patch being sampled. Each patch features an average local tilt that translates as shift of the spot focused by the respective microlens on the surface of its corresponding QC (Fig. 1b), that is, a quadrant detector composed of $2 \times 2$ photodiodes (PDs). Parameters of the WFS as the array grid, microlens size and material, have have been taken into account in order to more accurately estimate the resulting light spots on the sensor plane. The spot position on the surface of the $Q C$ determines the share of the spot intensity on each photodiode [18]. Therefore, the $x$ and $y$ spot-centroid coordinates can be related to the signals read from the QC photodiodes. The set of these coordinates throughout the array enables the estimation of the WF local $x$ and $y$ tilts that had caused the measured spot displacements. These registered tilts are then decomposed into mathematical functions, which in our case are Zernike polynomials. Each photodiode is a silicon $p-n$ junction that outputs a photocurrent proportional to the optical power impinging over its surface. It is modeled by an equivalent circuit as shown in Fig. 1c. A ROIC, either the APS or the Bouncing Pixel (Fig. 1d,e, respectively) [16, 19, 20], converts the current into an analog voltage that can be further converted to a digital number and stored for subsequent signal processing. Any limitation in the ROIC performance, therefore, directly impacts the accuracy of the reconstructed WF.

In Sect. 2, the Bouncing Pixel is introduced as an alternative to the APS as the readout circuit of an HS WFS. Section 3 presents the results for simulations for both circuit

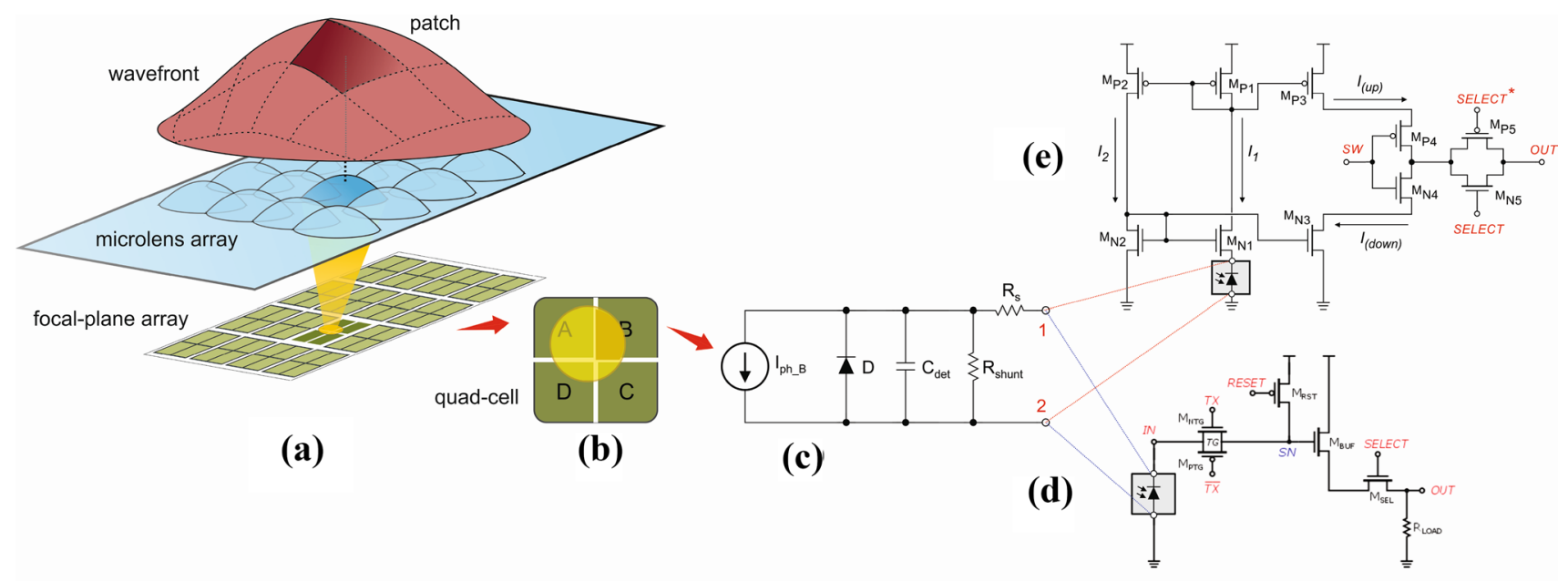

Fig. 1 a Schematic of a WFS for an input wavefront. b Quad-cell with light spot. c Photodiode model for the photodiode B of the quad-cell. d APS ROIC. e Bouncing-Pixel ROIC 
topologies, and their impact on the WFS final reconstruction error.

\section{The bouncing pixel}

As mentioned in the last section, each ROIC in a $Q C$ is responsible for transducing the photogenerated current from its respective PD to a proportional output voltage $V_{o}$ that can be further employed in calculations that will comprise the WF reconstruction. In some circuits $V_{o}$ decreases as the input current increases, and in some others it increases. In both cases, the more linear the relation between the output voltage and the input current a ROIC features, the better the circuit is, rendering compensation mechanisms unnecessary for signal linearization. Often, the topology is based on the photocurrent charging or discharging a capacitor over a certain pre-defined time interval, referred to as integration time $\Delta T$. The slope of the charging and discharging curve is proportional to the photocurrent magnitude. A steeper curve indicates a higher photocurrent. The voltage across the capacitor is then read at the end of the integration time and mimicked at the output by some chosen sort of electronic mirror, buffer or amplifier. In most cases, the pixel is then reset and a new integration and reading cycle begins.

The use of microlenses improves the overall signal-tonoise ratio (SNR) but consequently also increases the light intensity over each PD in the QC. The issue is that if a given WF patch impinging over a QC has an average slope that is too steep and leads most of the spot intensity towards one of the PDs, then the generated photocurrent might be too high and saturate the ROIC. This may also happen to any of the pixels if the light beam on the WFS is too intense. An optical neutral-density filter could avoid saturation but can both introduce additional aberrations and reduce the SNR, as has been reported before [21]. A usual constraint in circuit design that contributes to signal saturation of conventional integrated pixels is the maximum voltage allowed on chip $\left(V_{D D}\right)$, which is often no higher than about $3.3 \mathrm{~V}$ and decreases with the evolution of the CMOS processes towards smaller technology nodes, i.e. denser pixel integration. Alternative integrated pixel circuits that feature high dynamic range consistently compromise their signal sensitivity.

This trade-off between sensitivity and DR is observed in the several categories of circuits devised to widen the light-detection dynamic range of integrated pixels. One category consists of the Clipping Sensor, that uses capacitance changes, combining the sensitivity of different net capacitances $[22,23]$. It results in nonlinear response, lower SNR and reduced sensitivity for higher illuminations, when compared to architectures with moderate dynamic range. Another class comprises the Logarithmic Sensors that encompass circuits with a logarithmic output voltage response. They offer a substantial increase in the dynamic range, but also effect in continuously lowered sensitivity and SNR, especially as the illuminance level rises $[24,25]$. An additional approach is based on the Multimode Sensors that alternate between the logarithmic and linear modes, achieving HDR, with large SNR and sensitivity for low levels of illumination, when the linear mode is active, but undergoing the same described limitations for high illumination levels, for which the logarithmic mode is on $[26,27]$. Frequency-based Sensors constitute yet another categorey that converts the photocurrent into a related frequency pulse. They feature HDR and constant SNR, with a linear response. However, the level of the saturation current decreases as SNR increases $[24,28,29]$. The Time to First-Spike (TTS) Sensors monitor the required time for saturation to happen. By recording this figure and the saturation voltage, it is possible to reconstruct an HDR signal, although still featuring non-linearity and lower sensitivities for high illumination levels [24, 30-32]. Lastly, the categories Global-control-over-the-integrationtime Sensors [33, 34] and Autonomous-control-over-theintegration-time Sensors $[35,36]$ use a similar technique that employs multiple capture algorithms. The general scheme consists of integrating the same input photocurrent for two or more different integration time intervals $\Delta T$. By combining the responses of different capture intervals, it is possible to reconstruct an HDR signal. In spite of that, there the response is still non linear to a certain extent, the SNR dips and the sensitivity becomes lower for high illumination levels [24]. In summary, all the categories heretofore described present the same issues for achieving HDR: decreased sensitivity and SNR for high levels of illumination, SNR dips and response non-linearity; and none present an overall desired performance when implemented as integrated circuits $[15,24,30]$. Usually, off-the-shelf cameras, either based on CMOS or CCD image sensors, are employed as the focal-plane of HS wavefront sensors. Also, a number of custom VLSI solutions have been proposed for the focal plane of a wavefront sensor, featuring more direct spot-position detection by means of position-sensitive detector arrays and auxiliary electronics on chip [37-40]. None of them, however, have tackled the robustness to saturation under higher illumination levels along with the maintenance of high light-signal sensitivity and linearity.

To illustrate a situation where saturation can be detrimental, Fig. 2a shows an example where the light spot is evenly distributed over all photodiodes of a QC (A, B, $C$, and $D$ ), leading to equally generated photocurrents. The resulting output voltage $V_{o}$ for each ROIC is the value read after a certain integration time $\left(\Delta T_{1}\right)$, during which 

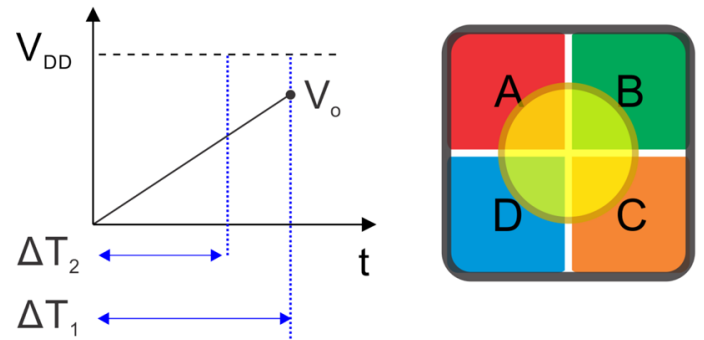

(a)
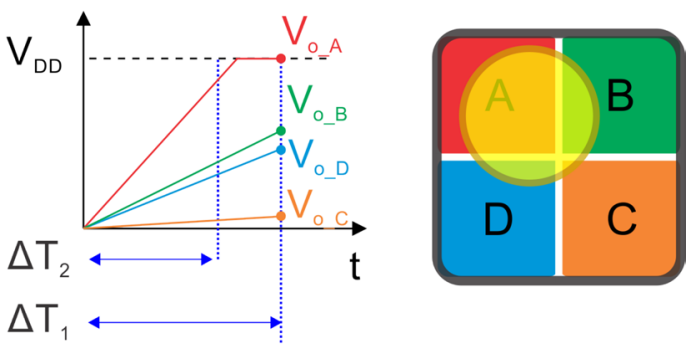

(b)

Fig. 2 Example QCs with different displacements of the light spot: a centered spot corresponding to a flat WF local patch; $\mathbf{b}$ a diagonally displaced spot corresponding to a tilted WF local patch

the voltage increases with time. All output voltages are equal and below the $V_{D D}$ upper limit. Figure $2 \mathrm{~b}$, however, presents the situation where a local tilt of the respective wavefront patch over the QC results in a spot shifted towards photodiode $A$. In this case, the voltage decreases for photodiodes $B, C$, and $D$, but increases drastically for photodiode $A$. As a result, the larger photocurrent produced may saturate the pixel output signal. Since the voltage $V_{o}$ feeds the calculation of spot coordinates and subsequently the WF reconstruction process, its saturation can severely impact the obtained output wavefront. A similar impact will occur in the detection of spot centroids on an orthogonal array of pixels in an image sensor, where the accuracy of the yielded centroid position may be affected by the number and position of saturated pixels illuminated by the spot. This issue becomes more probable as the pixel density increases, as smaller pixels, either in CCD or CMOS focal planes, tend to saturate for lower light intensities.

The merit function that measures the ability of a ROIC to avoid saturation is its input-current Dynamic Range (DR), that is related to the intensity of the input light $[29,41$, 42]. It consists of a relation in logarithmic scale between the maximum and minimum readable photocurrents for the ROIC. The APS is a simple ROIC, and its DR is usually not high, typically around $60 \mathrm{~dB}$, but can be improved by reducing the integration time, as for instance indicated by $\Delta T_{2}$ in the graphs of Fig. 2. In this case, for the ROIC reading the current from photodiode $A$, if the voltage is read right before it saturates, then unambiguous information is preserved. However, it comes at the cost of reducing the Sensitivity (S) [24], which is the ability to resolve two close output voltage values, therefore, to resolve two close input current values, affecting the resolution with which the centroid position of the light spot can be estimated. For instance, in $2 b$ the light spot has a slightly larger overlap with photodiode $B$ than with photodiode $D$, which would consequently produce a slightly larger voltage $V_{o}$ for ROIC B. Nonetheless, when the sensitivity is low, it might happen that the actual output voltage difference between $B$ and $D$ is so small that the circuit cannot distinguish between them. In summary, the larger the DR, the steeper are the slopes that can be measured in a WF, which increases the spatial resolution while sampling the WF. Also, the larger the $S$, the easier it is for the WFS to resolve two very close values of WF local slope, which reduces the reconstruction error. However, when deploying the APS as the chosen ROIC, there is an unavoidable trade-off between sensitivity and dynamic range, limiting the overall WFS performance either way.

The Bouncing Pixel, on the other hand, avoids saturation of the voltage signal by forcing mirrored currents to change the direction of integration when the signal would otherwise tend towards saturation $[15,16]$. It is based on a double current mirror, that delivers two currents that are equal in absolute value, but with opposite flowing directions. These currents are proportional to the photocurrent generated on the photodiode. Only one of these currents is conveniently selected at its turn and integrated on an external capacitor. When the integrated voltage reaches an upper threshold, the circuit selects the current that discharges the capacitor and, when it reaches the lower boundary, the circuit selects again the current that charges it. This process of swapping the photocurrent direction whenever the voltage signal reaches a predefined threshold value continues until the end of $\Delta T_{\text {BouncingPixel. }}$

Figure 3 illustrates the concept, comparing the APS (a) and the Bouncing Pixel (b), each for the same pair of equal values of input photocurrents. It can be seen that the APS needs to have a shorter integration time $\Delta T_{A P S}$ to be able to avoid saturation. This results in a smaller voltage difference at the output $\left(\delta V_{o}\right)$ for the same input current difference. This example illustrates both the increased DR of the Bouncing Pixel and its higher sensitivity.

The Bouncing Pixel employs a digital counter to mark the number of times the integrated voltage changes its direction. The counter has $b_{c}$ bits, which means it expands the virtual maximum supply voltage up to $V_{D D \text { (virtual) }}=2^{b_{c}} \cdot \Delta V$, that can be hundreds of times larger 


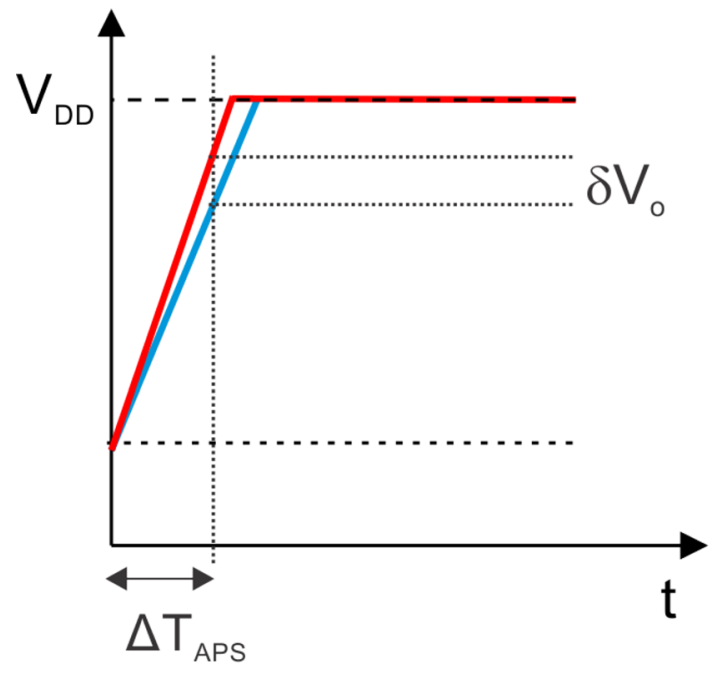

(a)

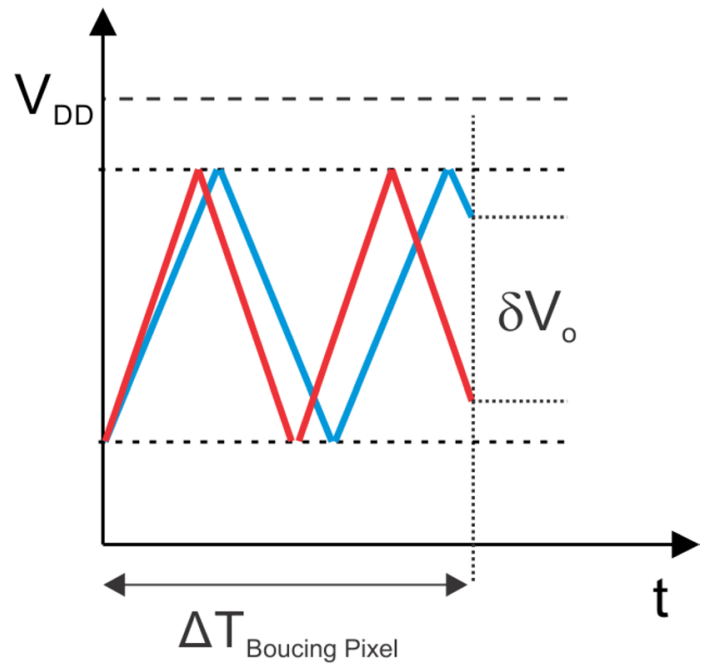

(b)

Fig. 3 Illustration of the output voltage for the a APS and the $\mathbf{b}$ bouncing pixel

than the actual $V_{D D}$. Since the sensitivity is actually the transimpedance gain throughout the input photocurrent span, it is given by $S=V_{D D \text { (virtual) }} / I_{\text {sat }}=\left(b_{c} . \Delta V\right) / I_{\text {sat }}$, where $I_{\text {sat }}$ is the maximum readable current before saturation, or the saturation current. The Dynamic Range, on the other hand, in a logarithmic scale, can be extended $\left(D R_{(e x t)}\right)$ by a factor equal to the number of times the saturation current can be increased [15]. The DR extension means that, if the pixel did not have the bouncing ability, like the APS, it would have a Dynamic Range of $D R_{\text {(base) }}$; however, due to its immunity to saturation, the bouncing pixel extended dynamic range is $D R_{(\text {total })}=D R_{(\text {base })}+D R_{(\text {ext })}$. In other words, for a given sensitivity, it is $D R_{(\text {ext })}=20 . \log \left(2^{b_{c}}\right)$, since for larger values of $b_{c^{\prime}} 2^{b_{c}}=V_{D D \text { (virtual) }} / \Delta V \approx V_{D D \text { (virtual) }} / V_{D D}$. Therefore, notice that for the Bouncing Pixel, with larger values of the virtual $V_{D D}$, both the DR and the $S$ can be simultaneously extended. For the APS, however, the virtual enhancement of $V_{D D}$ is not possible.

\section{Results}

In order to compare the performance of the WFS either with the APS or with the Bouncing Pixel, an input wavefront was employed based on the Porter statistics for the aberration of a population of human eyes [43]. This WF was fed into the computational platform, the wavefront was sampled and the photocurrents were calculated. The sampling plane of the HS was chosen as an orthogonal array with $N \times N$ microlenses, which determines the spatial resolution to sample the input WF. All simulations were performed for different numbers of microlenses in order to evaluate the impact of both the ROIC topologies and that of the sampling spatial frequencies on the final WF reconstruction errors. The simulations were carried out with two different input optical power levels $P_{i n}$, namely, $10 \mu W$ and $100 \mu W$. The input power is considered to be evenly distributed over the sampling area. This plane has a fixed lateral size and the more microlenses there are, the smaller each one is, and the lower the power of each focused light spot. In addition, it is important to realize that the employed electronic device models (BSIM3v3) are based on semi-empirical equations and are widely used to reliably predict circuits behavior during the design phase prior to fabrication in the microelectronics industry.

The merit function considered for comparison in this work is the WF RMS reconstruction error (in $\mu \mathrm{m}$ ) between the resulting reconstructed WF and the original one. In addition, all reconstruction-error results consider 3 different situations: a) Circumvention of the electronic circuitry, where the photocurrent values generated from the photodiodes in the QCs are directly used in the calculation of the spot coordinates; b) deployment of the Bouncing Pixel in the QCs translating the photocurrents into voltages; $c$ ) use of the APS, also transducing photocorrents to voltages. For the ROICs, the integration time is arbitrarily considered to be fixed at $\Delta T=10 \mathrm{~ms}$, which, for a the focal plane operating in the global shutter mode, and with a reset time of $1 \mathrm{~ms}$, would mean a WF sampling rate of approximately 90 $\mathrm{Hz}$. Furthermore, all simulations with either the Bouncing Pixel or the APS also consider the impact of noise in the output voltage. That was done by extracting the outputreferred noise from the power spectral distribution for each circuit, and by adding Gaussian noise to the output 
voltage. The results presented hereinafter show the average RMS error along with their standard deviation for a group of 30 different simulations for each ROIC and each density of the microlens array.

Figure 4a shows the RMS reconstruction error considering $P_{\text {in }}=10 \mu \mathrm{W}$ for different numbers of microlenses in the sampling array. It can be noticed from the graph that for 16 microlenses the RMS error for the APS is the largest one, whereas that for the Bouncing Pixel is much lower. Besides, the latter is conveniently almost the same as that without electronics. In addition, the standard deviation is much larger for the APS. For 25 microlenses, the errors for all situations are very close to each other. For 36 microlenses onwards, the error for the APS starts to diverge again. For the Bouncing Pixel, however, the error starts to diverge from that without electronics only for configurations with more than 49 microlenses. In conclusion, the trend presented in the graph shows that the Bouncing Pixel features an overall better performance than the APS, with lower RMS errors. Since less microlenses result in higher optical power levels over each QC, then for 16 microlenses the large error in the APS occurred due to saturation of its output voltage. The same does not happen for the Bouncing Pixel, since it features a much higher DR. For 25 microlenses, both ROICs feature similar RMS errors, as the APS is no longer saturated. However, for more microlenses, less power is shed over each QC, making it more difficult for the ROICs to distinguish between close photocurrents, therefore, between close local WF tilts. The Bouncing Pixel features a better precision on the WF reconstruction for larger arrays of microlenses due to its increased sensitivity, in comparison with the APS. In both cases, however, an increase in the reconstruction error is expected as the number of microlenses increase further, not only because of a lower SNR but also because the reconstruction matrix becomes larger and, consequently, errors introduced by the electronic noise-prone readings affect more elements throughout the intertwined numerical matrix calculations.

Figure $4 \mathrm{~b}$ shows the same sequence of simulations, but considering a higher $P_{\text {in }}=100 \mu \mathrm{W}$. It is clear that for the APS, the output was saturated, leading to a drastic increase in the error for all microlens dimensions, whereas the Bouncing Pixel follows a similar trend as that presented in Fig. 4a. In this figure, results are only shown up to 49 microlenses, solely due to simulation time, but it already proves that the tolerance to saturation, i.e. high DR, of the Bouncing Pixel indeed offers a benefit. For a larger number of microlenses, at that power level, the errors for the WFS with the APS are expected to drop as soon as the power proportionate to each microlens ceases to saturate the pixels in the respective QC.

Figure 5 shows the original WF (a) and the reconstructed WF with 49 microlensenses and $P_{\text {in }}=100 \mu W$, without electronics (b), with the APS (c) and with the Bouncing Pixel (d). The only WF that is substantially different from the original one is the WF reconstructed with the APS. It can be seen, as expected, that the residual WF for the APS (e) is much larger than that for the Bouncing Pixel (f).

\section{Discussion}

The results presented in the last section indicate the power and suitability of a flexible simulation platform in designing $\mathrm{AO}$ systems, particularly for the wavefront sensor, enabling not only the estimation of the overall system performance, but also the assessment of the impact single components may have. The platform enables changes in

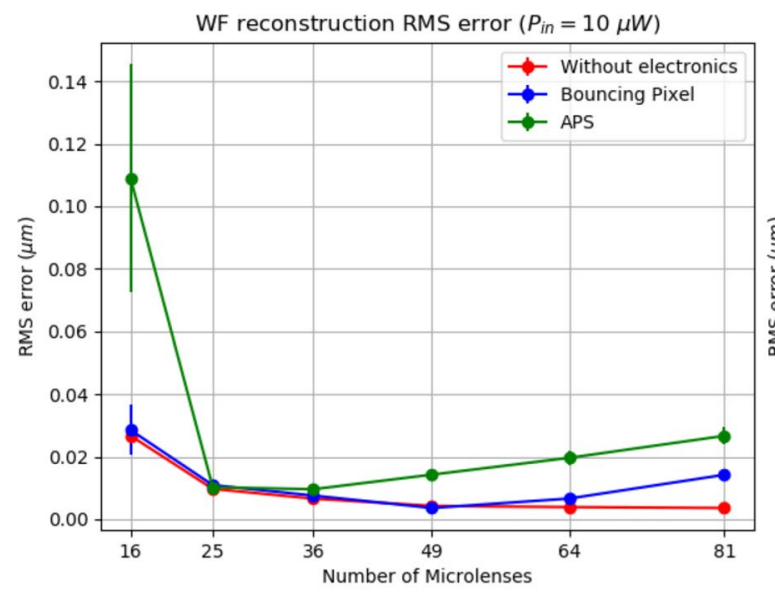

(a)

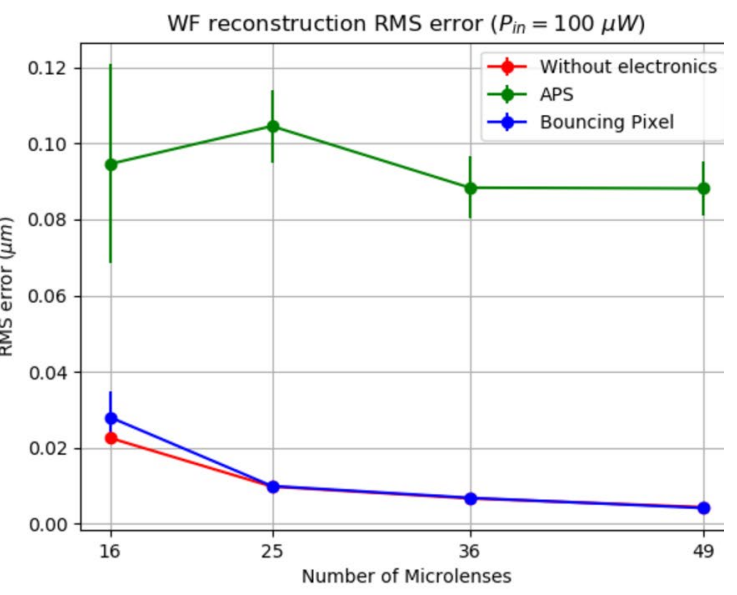

(b)

Fig. 4 Wavefront RMS reconstruction error for various numbers of microlenses for the APS and the Bouncing Pixel, compared to the situation withouth electronics, with an input power $\mathbf{a} P_{\text {in }}=10 \mu \mathrm{W}$ and $\mathbf{b} P_{\text {in }}=100 \mu \mathrm{W}$ 


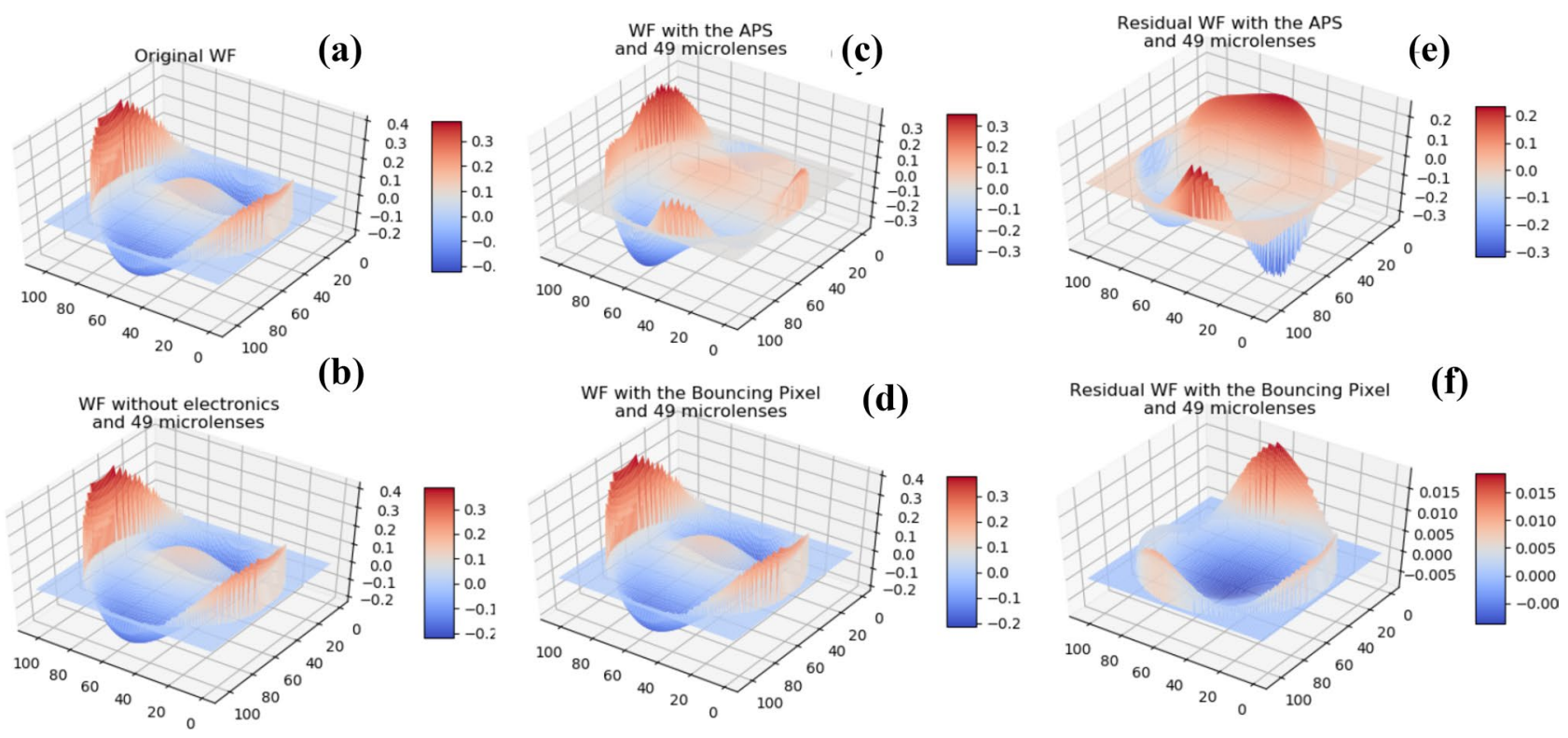

Fig. 5 Simulations with $P_{\text {in }}=100 \mu W$ for: a original WF; and the reconstructed WFs for 49 microlenses: $\mathbf{b}$ without electronics; c with the APS; and $\mathbf{d}$ with the Bouncing Pixel. The residual WFs are displayed for the case with $\mathbf{e}$ the APS; and $\mathbf{f}$ the Bouncing Pixel

the sensor architecture and components, such as the sampling plane and the discrete or integrated electronic sensors and circuits. This can assist in tailoring design choices to a specific application, and optimizing components prior to actual chip fabrication, reducing overall product costs and development time.

The results particularly show substantial benefits of employing the Bouncing Pixel instead of conventional read-out circuits for the focal-plane array, with preliminary comparison done to the APS. Its superior high dynamic range, linearity and sensitivity make its performance conveniently close to the optimal theoretical performance of a wavefront sensor without the interference of readout electronics. The Bouncing-Pixel circuit automatically renders the spot detection far more tolerant to illuminance saturation, while keeping its high sensitivity across a wide range of light levels. Besides, this pixel is not limited for use in the HS sensor and is suitable for deployment on the focal-plane array of other wavefront sensor types as well.

\section{Conclusions}

In this work, the Bouncing Pixel is investigated as a promising pixel topology to be deployed on the focal-plane array of a Hartmann-Shack wavefront sensor whose performance was evaluated using a custom simulation platform that integrates a circuit simulator with reliable integrated-device models of a running CMOS foundry. The platform models the whole system starting from the description of the original input wavefront, imparting a power density, sampling it by an orthogonal microlens array, employing refraction and projection of the spots on quad-cells, modelling photodetection on silicon pn junctions, converting photocurrents to voltages by means of readout integrated electronics, and reconstructing the wavefront. The system performance was evaluated by means of the RMS reconstruction error relative to the original input wavefront. The reconstruction results were compared with and without the impact of electronics, and for two distinct types of pixel readout circuits, namely the more conventional APS and the Bouncing Pixel; for different numbers of microlenses on the sampling plane, and for two different input optical power levels.

The Bouncing Pixel was custom designed to feature both extended dynamic range and high sensitivity. The comparisons showed that for a higher density of microlenses, where the optical power over each quad-cell is smaller, the large sensitivity of the Bouncing Pixel compared to that of the APS resulted in lower RMS errors, closer to the ideal situation without any circuit. Also, for fewer microlenses or for a higher optical power, the much larger dynamic range of the Bouncing Pixel yields a significantly better performance if compared to the APS. In conclusion, the results indicate that the Bouncing Pixel performs substantially better than the APS in all situations, introducing only minimal impact on the accuracy of reconstructed wavefront. 
Acknowledgements The authors are thankful to PPGEE (Graduate Program on Electrical Engineering - UFMG), FAPEMIG (Research Foundation of the State of Minas Gerais - Brazil), FAPEAM (Research Foundation of the State of Amazonas) and CNPq (National Research Council - Brazil) for institutional support and financial resources. This study was financed in part by the Coordenação de Aperfeiçoamento de Pessoal de Nível Superior - Brasil (CAPES) - Finance Code 001.

\section{Compliance with ethical standards}

Conflict of interest On behalf of all authors, the corresponding author states that there is no conflict of interest.

\section{References}

1. Tyson R (2012) Topics in adaptive optics. IntechOpen

2. Aftab M, Choi H, Liang R, Kim DW (2018) Adaptive shack-hartmann wavefront sensor accommodating large wavefront variations. Opt. Express 26(26):34428-34441

3. de Lima Monteiro DW, Vdovin G, Sarro PM (2004) High-speed wavefront sensor compatible with standard CMOS technology. Sens Actuators A: Phys 109(3):220-230

4. Basden AG, Bharmal NA, Jenkins D, Morris TJ, Osborn J, Peng J, Staykov $L$ (2018) The durham adaptive optics simulation platform (dasp): current status. SoftwareX 7:63-69

5. Reeves A (2016) Soapy: an adaptive optics simulation written purely in python for rapid concept development. In: Adaptive Optics Systems V, vol. 9909, p. 99097F. International Society for Optics and Photonics

6. Carbillet $M$, Vérinaud Ch, Femenía B, Riccardi A, Fini L (2005) Modelling astronomical adaptive optics-I. The software package caos. Monthly Not R Astron Soc 356(4):1263-1275

7. Wang L, Ellerbroek B (2011) Fast end-to-end multi-conjugate ao simulations using graphical processing units and the maos simulation code. Adapt Opt Extrem Large Telescopes II

8. Le Louarn M, Madec P-Y, Marchetti E, Bonnet H, Esselborn M (2016) Simulations of e-elt telescope effects on ao system performance. In: Adaptive optics systems V, vol 9909, p 990975. International Society for Optics and Photonics

9. Conan Rodolphe, Correia C (2014) Object-oriented matlab adaptive optics toolbox. In: Adaptive Optics Systems IV, vol. 9148, p. 91486C. International Society for Optics and Photonics

10. Ellerbroek BL (2005) Linear systems modeling of adaptive optics in the spatial-frequency domain. JOSA A 22(2):310-322

11. Jolissaint L, Véran J-P, Conan R (2006) Analytical modeling of adaptive optics: foundations of the phase spatial power spectrum approach. JOSA A 23(2):382-394

12. Rigaut F, Van Dam M (2013) Simulating astronomical adaptive optics systems using yao

13. Abecassis UV, de Lima Monteiro DW, Salles LP, Stanigher R, Borges E (2014) Computational test bench and flow chart for wavefront sensors. In: Optical modelling and design III, vol 9131, p 91312A. International Society for Optics and Photonics

14. Abecassis UV, de Lima Monteiro DW, Salles LP, de Moraes Cruz CA, Agra Belmonte PN (2018) Impact of cmos pixel and electronic circuitry in the performance of a Hartmann-Shack wavefront sensor. Sensors 18(10):3282

15. Belmonte PN, French PJ, de Lima Monteiro DW, Torres FS (2013) Linear high-dynamic-range bouncing pixel with single sample. In: Proceedings of 2013 international image sensor workshop, ISSW, pp 12-16

16. de Moraes Cruz CA, Belmonte PNA, de Lima Monteiro DW (2015) Linear mode high dynamic range bouncing pixel with single transistor. In: Proceedings of the international image sensors workshop, pp 1-4. IISW

17. Fossum ER, Mendis S, Kemeny SE (1995) Active pixel sensor with intra-pixel charge transfer, November 28. US Patent $5,471,515$

18. Salles LP, de Lima Monteiro DW (2009) Designing the response of an optical quad-cell as position-sensitive detector. IEEE Sens $\mathrm{J}$ 10(2):286-293

19. Retes PFL, Torres FS, de Lima Monteiro DW (2011) Evaluation of the full operational cycle of a cmos transfer-gated photodiode active pixel. Microelectron J 42(11):1269-1275

20. Costa LC, de Mello Artur SB, Salles LP, de Lima Monteiro DW (2015) Comparative analysis of $350 \mathrm{~nm} \mathrm{cmos}$ active pixel sensor electronics. In: 2015 30th Symposium on Microelectronics Technology and Devices (SBMicro), pp 1-4. IEEE

21. Hénault $F(2005)$ Wavefront sensor based on varying transmission filters: theory and expected performance. J Mod Opt 52(14):1917-1931

22. Decker S, McGrath D, Brehmer K, Sodini CG (1998) A 256/spl times $/ 256 \mathrm{cmos}$ imaging array with wide dynamic range pixels and column-parallel digital output. IEEE J Solid-state Circuits 33(12):2081-2091

23. Sugawa S, Akahane N, Adachi S, Mori K, Ishiuchi T, Mizobuchi $\mathrm{K}$ (2005) A $100 \mathrm{db}$ dynamic range cmos image sensor using a lateral overflow integration capacitor. In: ISSCC. 2005 IEEE international digest of technical papers. Solid-state circuits conference, 2005. pp 352-603. IEEE

24. Spivak A, Belenky A, Fish A, Yadid-Pecht O (2009) Wide-dynamicrange cmos image sensors-comparative performance analysis. IEEE Trans Electron Devices 56(11):2446-2461

25. Hagihara $Y$ (2001) Logarithmic-converting cmos area image sensors with an FPN cancel circuit. Kyokai Joho Imeji Zasshi 55(7):1039-1044

26. Akahane N, Ryuzaki R, Adachi S, Mizobuchi K, Sugawa S (2006) A $200 \mathrm{db}$ dynamic range iris-less cmos image sensor with lateral overflow integration capacitor using hybrid voltage and current readout operation. In: 2006 IEEE International solid state circuits conference-digest of technical papers, pp 1161-1170. IEEE

27. Fox EC, Hynecek J, Dykaar DR (2000) Wide-dynamic-range pixel with combined linear and logarithmic response and increased signal swing. In: Sensors and camera systems for scientific, industrial, and digital photography applications, vol 3965, pp 4-11. International Society for Optics and Photonics

28. Frohmader KP (1982) A novel mos compatible light intensityto-frequency converter suited for monolithic integration. IEEE J Solid-State Circuits 17(3):588-591

29. Wang $X$, Wong W, Hornsey $\mathrm{R}$ (2006) A high dynamic range cmos image sensor with inpixel light-to-frequency conversion. IEEE Trans Electron Devices 53(12):2988-2992

30. El Gamal A (2002) Ee392b classnotes: introduction to image sensors and digital cameras. ISSCC

31. Guo X, Qi X, Harris JG (2007) A time-to-first-spike cmos image sensor. IEEE Sens J 7(8):1165-1175

32. Brajovic V, Kanade T (1995) New massively parallel technique for global operations in embedded imagers. In: 1995 IEEE workshop on CCDs and advanced image sensors

33. Ambalathankandy P et al (2016) Real-time implementation of an exponent-based tone mapping algorithm. Ph.D. thesis, University of Calgary

34. Mase M, Kawahito S, Sasaki M, Wakamori Y, Furuta M (2005) A wide dynamic range cmos image sensor with multiple exposure-time signal outputs and 12-bit column-parallel cyclic a/d converters. IEEE J Solid-State Circuits 40(12):2787-2795

35. Hamamoto T, Aizawa K (2001) A computational image sensor with adaptive pixel-based integration time. IEEE J Solid-State Circuits 36(4):580-585 
36. Yadid-Pecht O, Belenky A (2003) In-pixel autoexposure cmos aps. IEEE J Solid-State Circuits 38(8):1425-1428

37. Monteiro DWL, Nirmaier T, Vdovin GV, Theuwissen AJP (2005) Fast Hartmann-Shack wavefront sensors manufactured in standard cmos technology. IEEE Sens J 5(5):976-982

38. Nirmaier T, Diez CA, Bille JF (2005) High-speed cmos wavefront sensor with resistive-ring networks of winner-take-all circuits. IEEE J Solid-state Circuits 40(11):2315-2322

39. Pui BH, Hayes-Gill B, Clark M, Somekh MG, See CW, Morgan S, $\mathrm{Ng} \mathrm{A}$ (2004) Integration of a photodiode array and centroid processing on a single cmos chip for a real-time Shack-Hartmann wavefront sensor. IEEE Sens J 4(6):787-794

40. McPhate J, Vallerga J, Tremsin A, Siegmund O, Mikulec B, Clark A (2005) Noiseless, kilohertz-frame-rate, imaging detector based on microchannel plates readout with the medipix $2 \mathrm{cmos}$ pixel chip. In: Infrared and photoelectronic imagers and detector devices, vol 5881, p 58810D. International Society for Optics and Photonics

41. Fossum ER (1997) Cmos image sensors: electronic camera-ona-chip. IEEE Trans Electron Devices 44(10):1689-1698

42. Yadid-Pecht O (1999) Wide-dynamic-range sensors. Opt Eng 38(10):1650-1660

43. Porter J, Guirao A, Cox IG, Williams DR (2001) Monochromatic aberrations of the human eye in a large population. JOSA A 18(8):1793-1803

Publisher's Note Springer Nature remains neutral with regard to jurisdictional claims in published maps and institutional affiliations. 